\title{
Theoretical Estimation of Grassmann's Transformation Resolution in Avionics Color Coding Systems
}

\author{
Yuri Armenakovich Gatchin ${ }^{1}$, Igor Olegovich Zharinov ${ }^{1}$, Anatoly Grigorevich Korobeynikov ${ }^{1} \&$ Oleg \\ Olegovich Zharinov ${ }^{2}$ \\ ${ }^{1}$ ITMO University, 49 Kronverksky Ave. St.Petersburg, Russia \\ ${ }^{2}$ SUAI, 67, Bolshaya Morskaia str., Saint-Petersburg, Russia \\ Correspondence: Anatoly Grigorevich Korobeynikov, ITMO University, 49 Kronverksky Ave. St.Petersburg, \\ 197101, Russia. Tel: 7-905-205-5262. E-mail: Korobeynikov_A_G@mail.ru
}

Received: January 22, 2015

Accepted: February 12, 2015

Online Published: March 25, 2015

doi:10.5539/mas.v9n5p197

URL: http://dx.doi.org/10.5539/mas.v9n5p197

\begin{abstract}
The problem of an assessment of the resolution of mathematical Grassmann's transformation, binding chromaticity coordinates in various systems of chromaticity coding, RGB and XY, is considered. Examples of graphic representations of colors and shades in various coding systems are given. The inference of the formula for an assessment of the resolution of Grassmann's transformation, specified as the minimal non-zero absolute value of $(x, y)$-chromaticity coordinates change, caused by any given $R G B$ code augmentation value, is proposed. Numerical values of the resolution are calculated, and combinations of the initial and final $R G B$ codes, which refer to the reached minimums of chromaticity coordinates changes, both $\Delta x$ and $\Delta y$, that caused by unit incrementation of the $R G B$ code, presuming eight-bit representation for each color component, $R, G, B$, are defined.
\end{abstract}

Keywords: Grassmann's transformation, chromaticity coding systems, resolution, systems of avionics

\section{Introduction}

Solutions of various avionic applied problems depends on research of physical principles and optical effects with the help of Grassmann's transformation (Barber, S., 2008; Kamoto, S., 2011). For example, problem of choosing relative parts of components of basic colors (red, green, blue).

The solution of a number of applied problems of modern aircraft instrumentation involves the study of physical principles and optical phenomena described in the language of mathematics with the Grassman's transformation. For such tasks, in particular, relates (Barber, S., 2008), the task of selecting the relative shares of the components of the primary colors (red, green, blue) components which form of the color palette of board means of display of navigation parameters and GIS data with enhanced visual characteristics of perception for humans.

Solution of the problem of colour palette selection for board display device involves the need for using an instrument that carries out modifications in selected color space. As practice and analysis of technical documentation for measuring equipment show, potential of modern colorimeters regarding resolution capability is limited, therefore the problem of search for theoretical estimate of resolution capability of Grassmann's transformation, which defines corresponding metrological characteristic of measuring instrument of trichromatic coordinates (chromaticity), is considered relevant.

Grassman's transformation introduces one-one correspondence numeric values (chromaticity coordinates) representing the same color (tint) in various coding systems. Aircraft display systems used a decimal system of color coding $R G B$ (Red, Green, Blue) for programming information display means, performed on the basis of the liquid crystal panel (Yamashita, J., 2014; Yoshida, Y., 2008), and the coding system $X Y$-to assess the quality of information visualization in different operating conditions of the equipment (the angles, the outer solar illumination etc).

Resolution of Grassmann's transformation subject to assessment defines the value of the minimum module discrete changes of $(x, y)$ chromaticity coordinates on the $X Y$-plane and generates a request to the appropriate metrological characteristics of the colorimeter to be used in full-scale experiments with onboard indicators. 
The problem of board display device colour palette components selection and solution of the problem of colour and brightness parameters calibration of LCD panels information display are relevant trends of development in modern colorimetry and optical instruments engineering. Studies in the area of the problem of selection of colour palette components of information display board device are represented by Kumar S.V., Ramana P.V. Mathematical rules of transition from one colour space to another are considered in detail by Ibraheem N.A., Hasan M.M., Khan R.Z., Mishra P.K.; Schanda J., 2007. Issues regarding development of soft hardware for display devices' visual characteristics calibration are addressed in scientific works by Seetzen H. and etc, 2007; Evanicky D. and etc, 2009.

Minimum value of discrete $(\Delta x, \Delta y)$ allows developers aviation equipment to determine the values of the decimal codes for RGB colors and shades with elevated values of contrast of the displayed image with the precision of a single increment code components of primary colors. Existing industrial designs measure of chromaticity coordinates have a resolution at the level $\Delta x \geq 0,02 \ldots 0,05 \quad \Delta y \geq 0,02 \ldots 0,05$ of units. Some samples of colorimeters, specially developed for research purposes, have a resolution of up to $0,002 \%$. These values do not provide the necessary developers onboard indicators of accuracy of selection relative shares of codes RGB color palette, and therefore must be defined special requirements to the characteristics of measuring instruments in assessing its resolution (Ishihara K., 2009; Zargaryants G. S., 2008).

\subsection{Grassmann's Transformation in Optical Color Coding Systems}

Correspondence between decimal $R G B$ code and $(x, y)$-coordinates of chromaticity on $X Y$-plane is based on Grassmann's (direct and inverse) transformations, which connect color code in basic color components $R G B \in[0$, 255] (Figure 1, a) with side values $X Y Z \in[0,1]$ of Maxwell's color triangle (Figure 1, b).

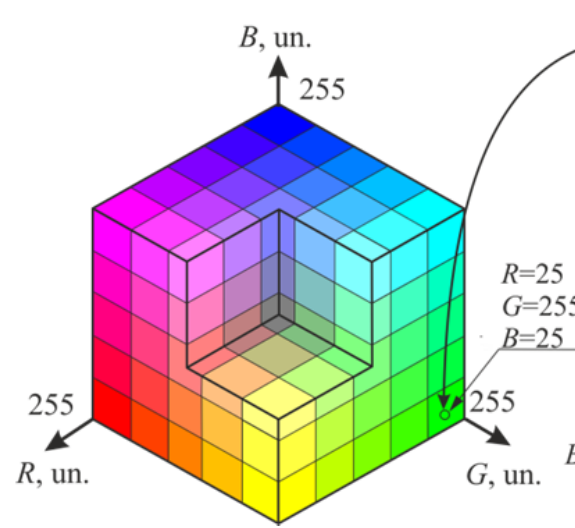

a)

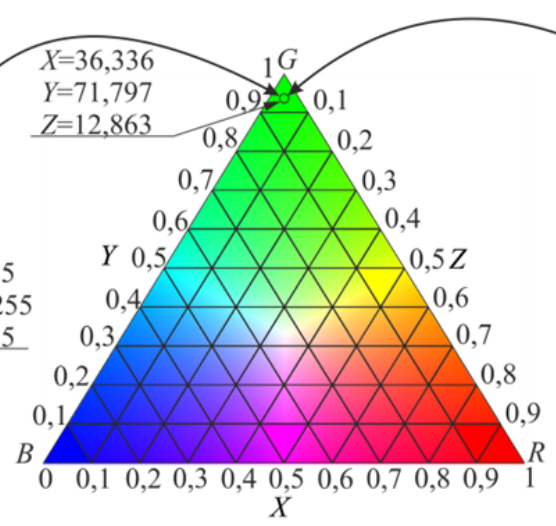

b)

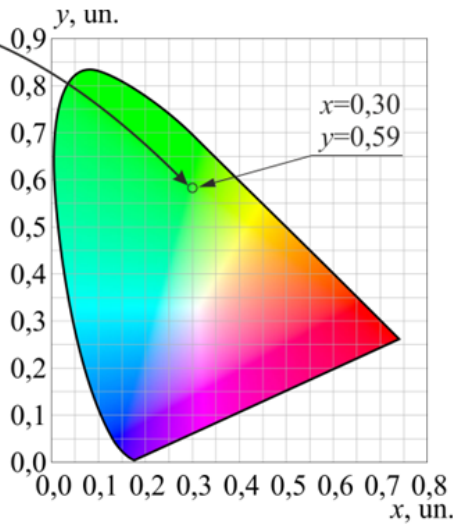

c)

Figure 1. Graphical representation of different color coding systems used in Grassmann's transformation: $R G B$; b) Maxwell's color triangle; c) $X Y$-plane.

Equations for direct $R G B \rightarrow X Y Z$ and inverse $X Y Z \rightarrow R G B$ Grassmann's transformation are as follows:

$$
\left[\begin{array}{c}
X \\
Y \\
Z
\end{array}\right]=\left[\begin{array}{lll}
X_{r} & X_{g} & X_{b} \\
Y_{r} & Y_{g} & Y_{b} \\
Z_{r} & Z_{g} & Z_{b}
\end{array}\right]\left[\begin{array}{l}
R \\
G \\
B
\end{array}\right],\left[\begin{array}{c}
R \\
G \\
B
\end{array}\right]=\left[\begin{array}{ccc}
X_{r} & X_{g} & X_{b} \\
Y_{r} & Y_{g} & Y_{b} \\
Z_{r} & Z_{g} & Z_{b}
\end{array}\right]^{-1}\left[\begin{array}{c}
X \\
Y \\
Z
\end{array}\right],
$$

where $X, Y, Z$ - color components in $X Y Z$ system; $X_{r}, X_{g}, X_{b}, Y_{r}, Y_{g}, Y_{b}, Z_{r}, Z_{g}, Z_{b}$ - color components determined used by CIE as etalon for color definition; $R, G, B$ - color code of main color components in decimal $R G B$ system. $X_{r}, Y_{r}, Z_{r}$ components determine rules of $R G B$ code transformation for etalon red color value, $X_{g}, Y_{g}, Z_{g}$ and $X_{b}, Y_{b}, Z_{b}$, components - for green and blue colors correspondingly.

Transition from side values of $X Y Z$ Maxwell's triangle to chromaticity $(x, y)$ - coordinates (Figure 1, c) of image elements can be done with the help of equations:

$$
x=\frac{X}{X+Y+Z}, \quad y=\frac{Y}{X+Y+Z}, \quad z=\frac{Z}{X+Y+Z},
$$

$z$ coordinate may not be calculated in this transition. 
Example of navigational frame of onboard indication device generated with $R G B$ and $(x, y)$ components is shown in Figure 2.

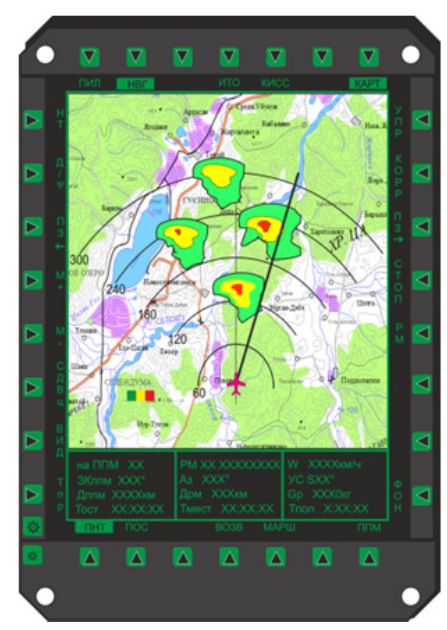

Figure 2. Example of navigational frame of onboard indication device

\section{Theoretical Estimation of Grassmann's Transformation Resolution}

As analysis (1), (2) shows every discrete increment of $R G B$ code, increases $X Y$-plane coordinates $\Delta x=x_{i+1}-x_{i}$, $\Delta y=y_{i+1}-y_{i}$. These increments values can be evaluated like that.

In accordance with (1), (2):

$$
\begin{gathered}
\left\{\begin{array}{l}
\Delta x=\left.x_{i+1}\right|_{R_{i+1} G_{i+1} B_{i+1}}-\left.x_{i}\right|_{R_{i} G_{i} B_{i}}=\frac{X_{i+1}}{X_{i+1}+Y_{i+1}+Z_{i+1}}-\frac{X_{i}}{X_{i}+Y_{i}+Z_{i}} \\
\Delta y=\left.y_{i+1}\right|_{R_{i+1} G_{i+1} B_{i+1}}-\left.y_{i}\right|_{R_{i} G_{i} B_{i}}=\frac{Y_{i+1}}{X_{i+1}+Y_{i+1}+Z_{i+1}}-\frac{Y_{i}}{X_{i}+Y_{i}+Z_{i}}
\end{array}\right. \\
\Rightarrow\left\{\begin{array}{l}
\Delta x=\frac{X_{i+1} Y_{i}+X_{i+1} Z_{i}-X_{i} Y_{i+1}-X_{i} Z_{i+1}}{\left(X_{i}+Y_{i}+Z_{i}\right)\left(X_{i+1}+Y_{i+1}+Z_{i+1}\right)} \\
\Delta y=\frac{Y_{i+1} X_{i}+Y_{i+1} Z_{i}-Y_{i} X_{i+1}-Y_{i} Z_{i+1}}{\left(X_{i}+Y_{i}+Z_{i}\right)\left(X_{i+1}+Y_{i+1}+Z_{i+1}\right)}
\end{array}\right.
\end{gathered}
$$

where

$$
\begin{gathered}
X_{i}=X_{r} R_{i}+X_{g} G_{i}+X_{b} B_{i}, \quad Y_{i}=Y_{r} R_{i}+Y_{g} G_{i}+Y_{b} B_{i}, \\
Z_{i}=Z_{r} R_{i}+Z_{g} G_{i}+Z_{b} B_{i}, \quad X_{i+1}=X_{r} R_{i+1}+X_{g} G_{i+1}+X_{b} B_{i+1}, \\
Y_{i+1}=Y_{r} R_{i+1}+Y_{g} G_{i+1}+Y_{b} B_{i+1}, \quad Z_{i+1}=Z_{r} R_{i+1}+Z_{g} G_{i+1}+Z_{b} B_{i+1} .
\end{gathered}
$$

After change of variables and removal of brackets numerators in first and second equation in system (3) will look like:

$$
\begin{gathered}
X_{i+1} Y_{i}+X_{i+1} Z_{i}-X_{i} Y_{i+1}-X_{i} Z_{i+1}= \\
=\left(R_{i+1} G_{i}-R_{i} G_{i+1}\right)\left(X_{r} Y_{g}+X_{r} Z_{g}-X_{g} Y_{r}-X_{g} Z_{r}\right)+ \\
+\left(R_{i+1} B_{i}-R_{i} B_{i+1}\right)\left(X_{r} Y_{b}+X_{r} Z_{b}-X_{b} Y_{r}-X_{b} Z_{r}\right)+
\end{gathered}
$$




$$
\begin{aligned}
& +\left(G_{i+1} B_{i}-G_{i} B_{i+1}\right)\left(X_{g} Y_{b}+X_{g} Z_{b}-X_{b} Y_{g}-X_{b} Z_{g}\right)=
\end{aligned}
$$

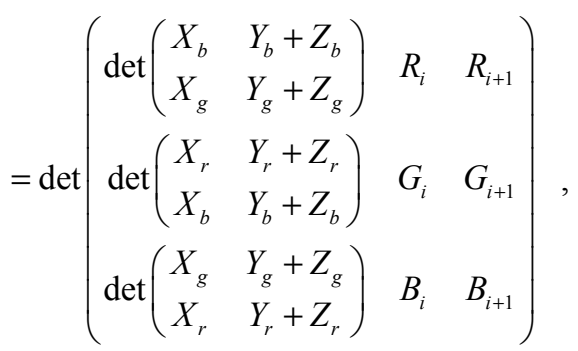

$$
\begin{aligned}
& Y_{i+1} X_{i}+Y_{i+1} Z_{i}-Y_{i} X_{i+1}-Y_{i} Z_{i+1}= \\
& =\left(R_{i+1} G_{i}-R_{i} G_{i+1}\right)\left(Y_{r} X_{g}+Y_{r} Z_{g}-Y_{g} X_{r}-Y_{g} Z_{r}\right)+ \\
& +\left(R_{i+1} B_{i}-R_{i} B_{i+1}\right)\left(Y_{r} X_{b}+Y_{r} Z_{b}-Y_{b} X_{r}-Y_{b} Z_{r}\right)+ \\
& +\left(G_{i+1} B_{i}-G_{i} B_{i+1}\right)\left(Y_{g} X_{b}+Y_{g} Z_{b}-Y_{b} X_{g}-Y_{b} Z_{g}\right)=
\end{aligned}
$$

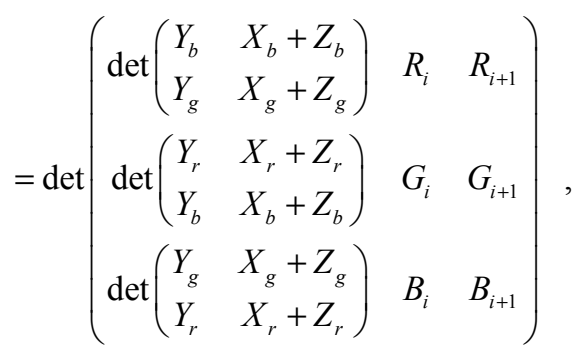

At the final step of the expressions (4), (5) the rule applies decomposition of the determinant by the first column and minors. The denominator of the equations of system (3) is equal to:

$$
\begin{gathered}
\left(X_{i}+Y_{i}+Z_{i}\right)\left(X_{i+1}+Y_{i+1}+Z_{i+1}\right)= \\
=\left(R_{i}\left(X_{r}+Y_{r}+Z_{r}\right)+G_{i}\left(X_{g}+Y_{g}+Z_{g}\right)+B_{i}\left(X_{b}+Y_{b}+Z_{b}\right)\right) \times \\
\times\left(R_{i+1}\left(X_{r}+Y_{r}+Z_{r}\right)+G_{i+1}\left(X_{g}+Y_{g}+Z_{g}\right)+B_{i+1}\left(X_{b}+Y_{b}+Z_{b}\right)\right)= \\
=\left(a_{r} R_{i}+a_{g} G_{i}+a_{b} B_{i}\right)\left(a_{r} R_{i+1}+a_{g} G_{i+1}+a_{b} B_{i+1}\right),
\end{gathered}
$$

where $a_{r}=X_{r}+Y_{r}+Z_{r}, a_{g}=X_{g}+Y_{g}+Z_{g}, a_{b}=X_{b}+Y_{b}+Z_{b}$.

Thereby increment values of $\Delta x=x_{i+1}-x_{i}, \Delta y=y_{i+1}-y_{i}$ coordinates of $X Y$-plane, corresponding to discrete change of decimal $R G B \in[0,255]$ code from $R_{i} G_{i} B_{i}$ value to $R_{i+1} G_{i+1} B_{i+1}$, value are: 


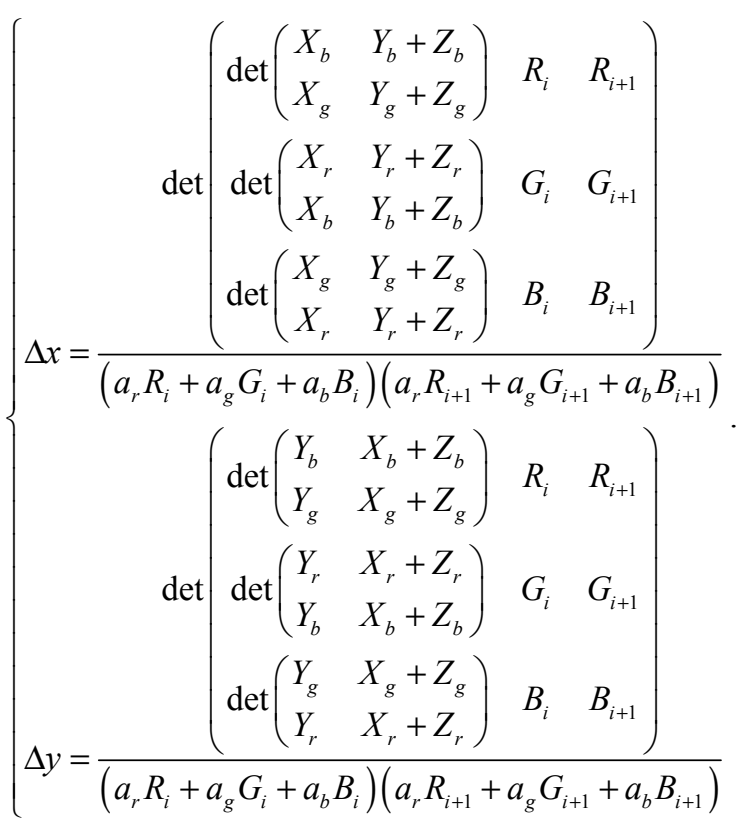

Combination codes $R=G=B=0$ combination, which stands for black color is not used for determining the start $R_{i} G_{i} B_{i}$ value. Minimal starting $R_{i} G_{i} B_{i}$ code values are decimal combinations $[0,0,1],[0,1,0]$ or $[1,0,0]$, where at least one of the values is not equal to zero.

Code increment

$$
R_{i+1}=R_{i}+\Delta R, G_{i+1}=G_{i}+\Delta G, B_{i+1}=B_{i}+\Delta B,
$$

can be implemented independently (separately, i.e. $\Delta R \neq \Delta G \neq \Delta B$, or simultaneously, i.e. $\Delta R=\Delta G=\Delta B$ ) on all three color components with following resolution $\Delta R \in[1,2, \ldots, 255], \Delta G \in[1,2, \ldots, 255], \Delta B \in[1,2, \ldots, 255]$, where: $1 \leq R_{i+1} \leq 255,1 \leq G_{i+1} \leq 255,1 \leq B_{i+1} \leq 255$.

To calculate $(\Delta x, \Delta y)$ values in numerators of system (6) equationsлях $R_{i+1}, G_{i+1}, B_{i+1}$ code values in the third matrix determinant column can be changed for corresponding increments $\Delta R, \Delta G, \Delta B$ of basic color components. Minimal increment step for each color component is: $\Delta R_{\min }=\Delta G_{\min }=\Delta B_{\min }=1$.

Increment modules $|\Delta x|,|\Delta y|$, calculated with the help of equation system (6), show that minimal step of $|\Delta x|$ value equals $8,45 \cdot 10^{-10}$ and can be achieved while moving from $R G B$ code point $(7,221,254)$ to point $(7,222$, 255). Minimal step of $|\Delta y|$ value equals $7,23 \cdot 10^{-10}$ and can be achieved while moving from $R G B$ code point $(43,254,47)$ to point $(44,255,47)$. Diagonal transfer $\sqrt{\Delta x^{2}+\Delta y^{2}}$ has minimum on $X Y$-plane with $1,614 \cdot 10^{-6}$ value and can be achieved while moving from $R G B$ code point $(253,254,0)$ to point $(254,255,0)$. Minimums of horizontal, vertical and diagonal increments were obtained in vicinities of different $R G B$ code values.

\section{Resolution of Grassmann's Transformation in Extremum Points}

Grassmann's transformation resolution in extremum points can be evaluated by substitution of (7) to (6), calculation of partial finite difference ratio $\Delta x: \partial \Delta x(\Delta R, \Delta G, \Delta B) / \partial \Delta R, \partial \Delta x(\Delta R, \Delta G, \Delta B) / \partial \Delta G$, $\partial \Delta x(\Delta R, \Delta G, \Delta B) / \partial \Delta B ; \quad \Delta y: \partial \Delta y(\Delta R, \Delta G, \Delta B) / \partial \Delta R, \partial \Delta y(\Delta R, \Delta G, \Delta B) / \partial \Delta G, \partial \Delta y(\Delta R, \Delta G, \Delta B) / \partial \Delta B$ and setting these ratios equal to 0 with subsequent solution of resulting system relatively to $\Delta R, \Delta G, \Delta B$.

Due to the fractional-rational view of the right part of expression (6), to simplify the analytical calculations when deriving the relations of finite difference it is advisable to use the rule of «logarithmic differentiation» the left and right expressions. The final expressions for the relations of finite differences in analytical form are of the form: 


$$
\begin{aligned}
& =\Delta G \frac{a_{g} \operatorname{det}\left(\begin{array}{cc}
G_{i} & \operatorname{det}\left(\begin{array}{ll}
X_{b} & Y_{b}+Z_{b} \\
X_{r} & Y_{r}+Z_{r}
\end{array}\right) \\
B_{i} & \operatorname{det}\left(\begin{array}{ll}
X_{r} & Y_{r}+Z_{r} \\
X_{g} & Y_{g}+Z_{g}
\end{array}\right)
\end{array}\right)+a_{r} \operatorname{det}\left(\begin{array}{ll}
R_{i} & \operatorname{det}\left(\begin{array}{cc}
X_{g} & Y_{g}+Z_{g} \\
X_{b} & Y_{b}+Z_{b}
\end{array}\right) \\
B_{i} & \operatorname{det}\left(\begin{array}{cc}
X_{r} & Y_{r}+Z_{r} \\
X_{g} & Y_{g}+Z_{g}
\end{array}\right)
\end{array}\right)}{\left(a_{r} R_{i}+a_{g} G_{i}+a_{b} B_{i}\right)\left(a_{r}\left(R_{i}+\Delta R\right)+a_{g}\left(G_{i}+\Delta G\right)+a_{b}\left(B_{i}+\Delta B\right)\right)^{2}}+
\end{aligned}
$$

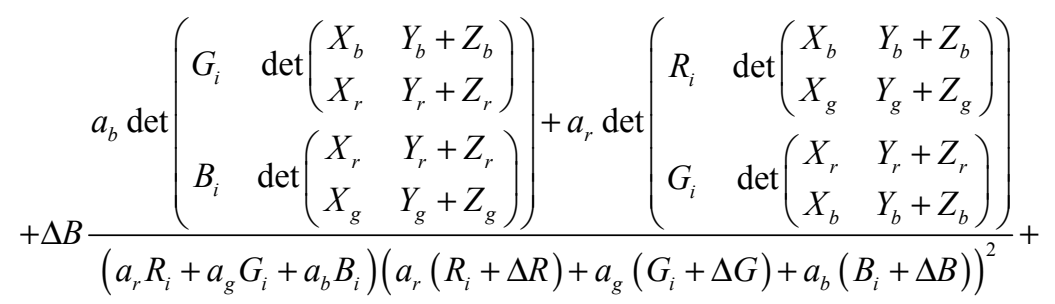

$$
\begin{aligned}
& +\frac{\operatorname{det}\left(\begin{array}{cc}
G_{i} & \operatorname{det}\left(\begin{array}{cc}
X_{b} & Y_{b}+Z_{b} \\
X_{r} & Y_{r}+Z_{r}
\end{array}\right) \\
B_{i} & \operatorname{det}\left(\begin{array}{ll}
X_{r} & Y_{r}+Z_{r} \\
X_{g} & Y_{g}+Z_{g}
\end{array}\right)
\end{array}\right)}{\left(a_{r}\left(R_{i}+\Delta R\right)+a_{g}\left(G_{i}+\Delta G\right)+a_{b}\left(B_{i}+\Delta B\right)\right)^{2}} .
\end{aligned}
$$

2. $\partial \Delta x(\Delta R, \Delta G, \Delta B) / \partial \Delta G=$

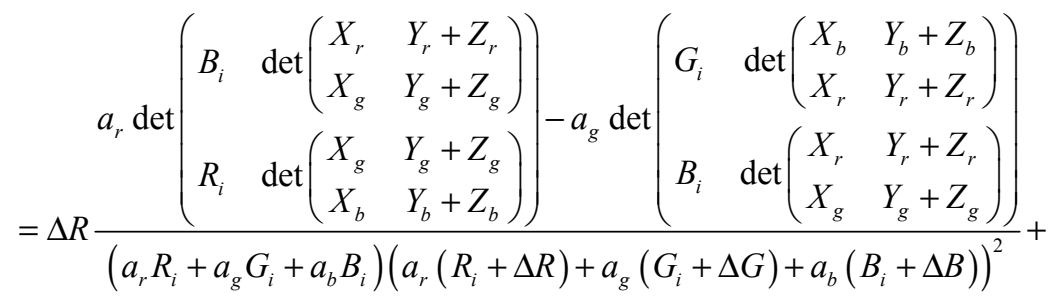

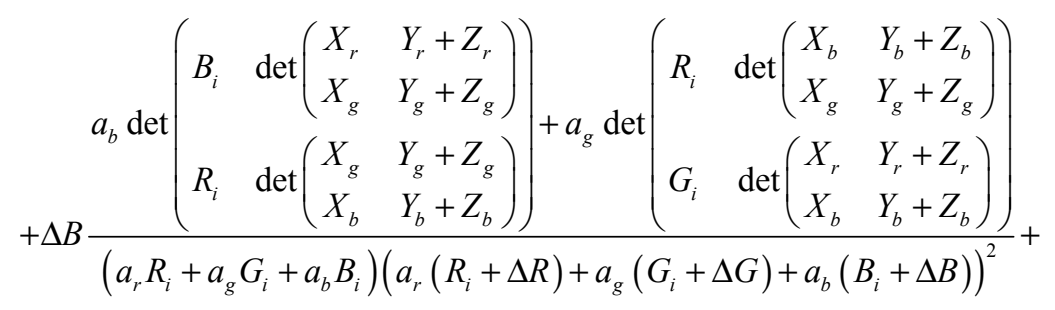

$$
\begin{aligned}
& +\frac{\operatorname{det}\left(\begin{array}{cc}
B_{i} & \operatorname{det}\left(\begin{array}{cc}
X_{r} & Y_{r}+Z_{r} \\
X_{b} & Y_{b}+Z_{b}
\end{array}\right) \\
R_{i} & \operatorname{det}\left(\begin{array}{cc}
X_{g} & Y_{g}+Z_{g} \\
X_{b} & Y_{b}+Z_{b}
\end{array}\right)
\end{array}\right)}{\left(a_{r}\left(R_{i}+\Delta R\right)+a_{g}\left(G_{i}+\Delta G\right)+a_{b}\left(B_{i}+\Delta B\right)\right)^{2}} .
\end{aligned}
$$

3. $\partial \Delta x(\Delta R, \Delta G, \Delta B) / \partial \Delta B=$ 


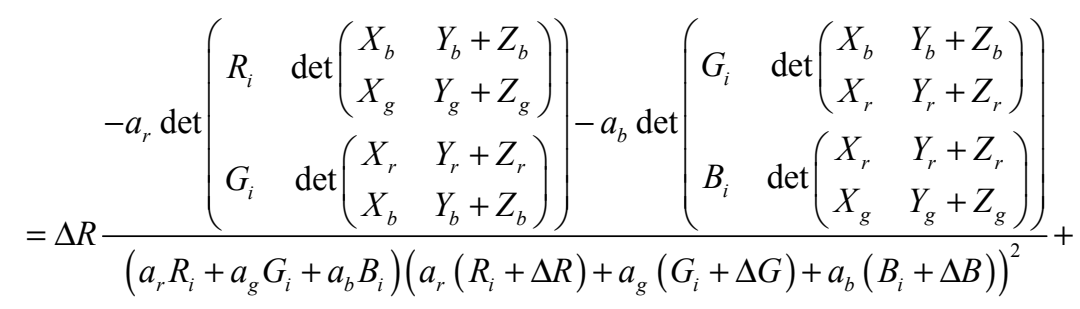

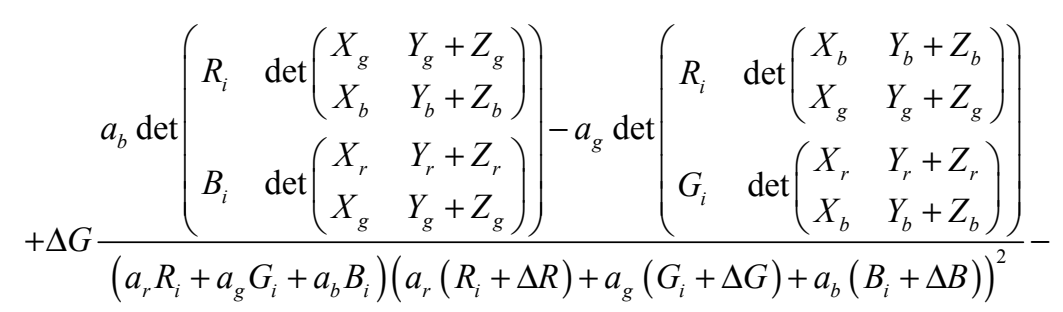

$$
\begin{aligned}
& -\frac{\operatorname{det}\left(\begin{array}{cc}
R_{i} & \operatorname{det}\left(\begin{array}{cc}
X_{b} & Y_{b}+Z_{b} \\
X_{g} & Y_{g}+Z_{g}
\end{array}\right) \\
G_{i} & \operatorname{det}\left(\begin{array}{ll}
X_{r} & Y_{r}+Z_{r} \\
X_{b} & Y_{b}+Z_{b}
\end{array}\right)
\end{array}\right)}{\left(a_{r}\left(R_{i}+\Delta R\right)+a_{g}\left(G_{i}+\Delta G\right)+a_{b}\left(B_{i}+\Delta B\right)\right)^{2}} .
\end{aligned}
$$

4. $\partial \Delta y(\Delta R, \Delta G, \Delta B) / \partial \Delta R=$

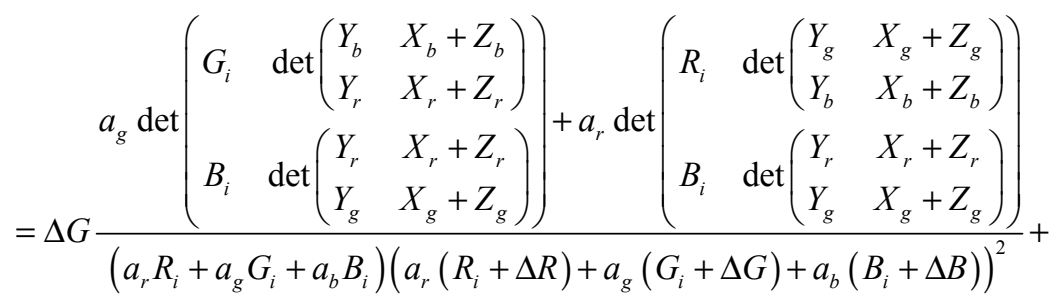

$$
\begin{aligned}
& +\Delta B \frac{a_{b} \operatorname{det}\left(\begin{array}{ll}
G_{i} & \operatorname{det}\left(\begin{array}{ll}
Y_{b} & X_{b}+Z_{b} \\
Y_{r} & X_{r}+Z_{r}
\end{array}\right) \\
B_{i} & \operatorname{det}\left(\begin{array}{ll}
Y_{r} & X_{r}+Z_{r} \\
Y_{g} & X_{g}+Z_{g}
\end{array}\right)
\end{array}\right)+a_{r} \operatorname{det}\left(\begin{array}{ll}
R_{i} & \operatorname{det}\left(\begin{array}{ll}
Y_{b} & X_{b}+Z_{b} \\
Y_{g} & X_{g}+Z_{g}
\end{array}\right) \\
G_{i} & \operatorname{det}\left(\begin{array}{ll}
Y_{r} & X_{r}+Z_{r} \\
Y_{b} & X_{b}+Z_{b}
\end{array}\right)
\end{array}\right)}{\left(a_{r} R_{i}+a_{g} G_{i}+a_{b} B_{i}\right)\left(a_{r}\left(R_{i}+\Delta R\right)+a_{g}\left(G_{i}+\Delta G\right)+a_{b}\left(B_{i}+\Delta B\right)\right)^{2}}+ \\
& +\frac{\operatorname{det}\left(\begin{array}{ll}
G_{i} & \operatorname{det}\left(\begin{array}{cc}
Y_{b} & X_{b}+Z_{b} \\
Y_{r} & X_{r}+Z_{r}
\end{array}\right) \\
B_{i} & \operatorname{det}\left(\begin{array}{cc}
Y_{r} & X_{r}+Z_{r} \\
Y_{g} & X_{g}+Z_{g}
\end{array}\right)
\end{array}\right)}{\left(a_{r}\left(R_{i}+\Delta R\right)+a_{g}\left(G_{i}+\Delta G\right)+a_{b}\left(B_{i}+\Delta B\right)\right)^{2}} .
\end{aligned}
$$

5. $\partial \Delta y(\Delta R, \Delta G, \Delta B) / \partial \Delta G=$ 


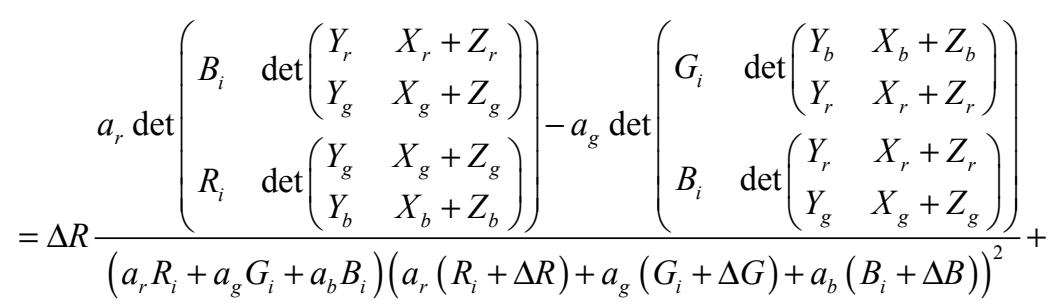

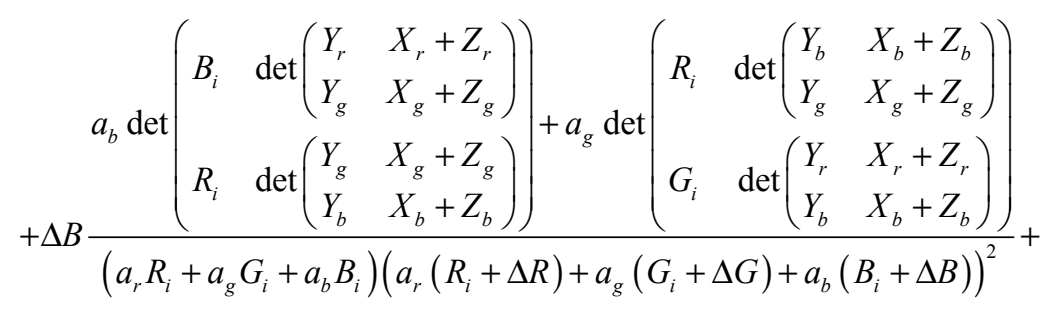

$$
\begin{aligned}
& +\frac{\operatorname{det}\left(\begin{array}{cc}
B_{i} & \operatorname{det}\left(\begin{array}{cc}
Y_{r} & X_{r}+Z_{r} \\
Y_{b} & X_{b}+Z_{b}
\end{array}\right) \\
R_{i} & \operatorname{det}\left(\begin{array}{cc}
Y_{g} & X_{g}+Z_{g} \\
Y_{b} & X_{b}+Z_{b}
\end{array}\right)
\end{array}\right)}{\left(a_{r}\left(R_{i}+\Delta R\right)+a_{g}\left(G_{i}+\Delta G\right)+a_{b}\left(B_{i}+\Delta B\right)\right)^{2}} .
\end{aligned}
$$

6. $\partial \Delta y(\Delta R, \Delta G, \Delta B) / \partial \Delta B=$

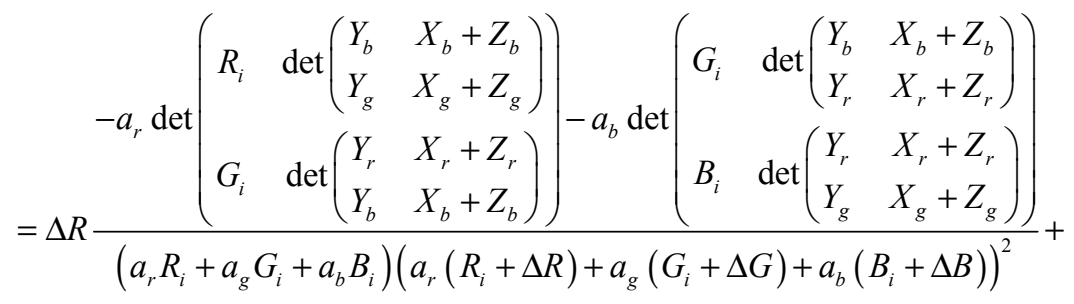

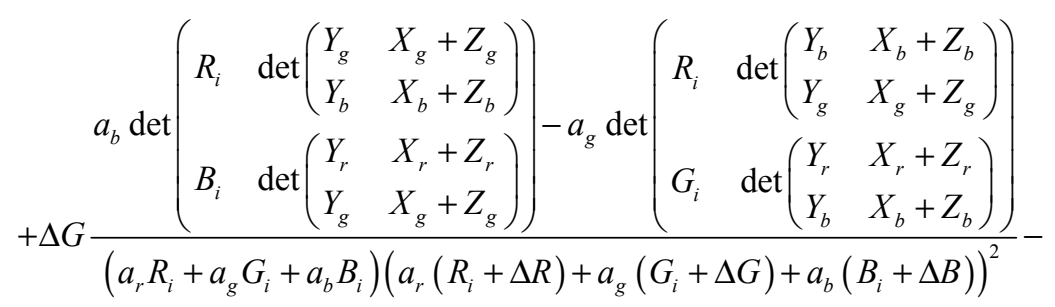

$$
\begin{aligned}
& -\frac{\operatorname{det}\left(\begin{array}{cc}
R_{i} & \operatorname{det}\left(\begin{array}{cc}
Y_{b} & X_{b}+Z_{b} \\
Y_{g} & X_{g}+Z_{g}
\end{array}\right) \\
G_{i} & \left.\operatorname{det}\left(\begin{array}{cc}
Y_{r} & X_{r}+Z_{r} \\
Y_{b} & X_{b}+Z_{b}
\end{array}\right)\right)
\end{array}\right)}{\left(a_{r}\left(R_{i}+\Delta R\right)+a_{g}\left(G_{i}+\Delta G\right)+a_{b}\left(B_{i}+\Delta B\right)\right)^{2}} .
\end{aligned}
$$

Search increments $\left\{\Delta R_{\Delta x}, \Delta G_{\Delta x}, \Delta B_{\Delta x}\right\},\left\{\Delta R_{\Delta y}, \Delta G_{\Delta y}, \Delta B_{\Delta y}\right\}$ in points, where is achieved extr $(\Delta x)$, $\operatorname{extr}(\Delta y)$, accomplished by solving systems: 


$$
\left\{\begin{array}{l}
\frac{\partial \Delta x(\Delta R, \Delta G, \Delta B)}{\partial \Delta R}=0 \\
\frac{\partial \Delta x(\Delta R, \Delta G, \Delta B)}{\partial \Delta G}=0 \\
\frac{\partial \Delta x(\Delta R, \Delta G, \Delta B)}{\partial \Delta B}=0
\end{array},\left\{\begin{array}{l}
\frac{\partial \Delta y(\Delta R, \Delta G, \Delta B)}{\partial \Delta R}=0 \\
\frac{\partial \Delta y(\Delta R, \Delta G, \Delta B)}{\partial \Delta G}=0 \\
\frac{\partial \Delta y(\Delta R, \Delta G, \Delta B)}{\partial \Delta B}=0
\end{array} .\right.\right.
$$

After substituting (8)-(13), (14) and bring the systems of equations (14) to the matrix species separately to $x$-coordinate and separately for the $y$-coordinate expression for both systems (14) can be rewritten:

$$
\left[\begin{array}{ccc}
0 & M_{12} & M_{13} \\
M_{21} & 0 & M_{23} \\
M_{31} & M_{32} & 0
\end{array}\right]\left[\begin{array}{l}
\Delta R_{\Delta x} \\
\Delta G_{\Delta x} \\
\Delta B_{\Delta x}
\end{array}\right]=\left[\begin{array}{c}
L_{1} \\
L_{2} \\
L_{3}
\end{array}\right],\left[\begin{array}{ccc}
0 & N_{12} & N_{13} \\
N_{21} & 0 & N_{23} \\
N_{31} & N_{32} & 0
\end{array}\right]\left[\begin{array}{l}
\Delta R_{\Delta y} \\
\Delta G_{\Delta y} \\
\Delta B_{\Delta y}
\end{array}\right]=\left[\begin{array}{c}
K_{1} \\
K_{2} \\
K_{3}
\end{array}\right],
$$

where

$$
\begin{aligned}
& L_{1}=-\left(a_{r} R_{i}+a_{g} G_{i}+a_{b} B_{i}\right) \operatorname{det}\left(\begin{array}{ll}
G_{i} & \operatorname{det}\left(\begin{array}{cc}
X_{b} & Y_{b}+Z_{b} \\
X_{r} & Y_{r}+Z_{r}
\end{array}\right) \\
B_{i} & \operatorname{det}\left(\begin{array}{ll}
X_{r} & Y_{r}+Z_{r} \\
X_{g} & Y_{g}+Z_{g}
\end{array}\right)
\end{array}\right), \\
& L_{2}=-\left(a_{r} R_{i}+a_{g} G_{i}+a_{b} B_{i}\right) \operatorname{det}\left(\begin{array}{cc}
B_{i} & \operatorname{det}\left(\begin{array}{cc}
X_{r} & Y_{r}+Z_{r} \\
X_{b} & Y_{b}+Z_{b}
\end{array}\right) \\
R_{i} & \operatorname{det}\left(\begin{array}{ll}
X_{g} & Y_{g}+Z_{g} \\
X_{b} & Y_{b}+Z_{b}
\end{array}\right)
\end{array}\right), \\
& L_{3}=\left(a_{r} R_{i}+a_{g} G_{i}+a_{b} B_{i}\right) \operatorname{det}\left(\begin{array}{ll}
R_{i} & \operatorname{det}\left(\begin{array}{ll}
X_{b} & Y_{b}+Z_{b} \\
X_{g} & Y_{g}+Z_{g}
\end{array}\right) \\
G_{i} & \operatorname{det}\left(\begin{array}{ll}
X_{r} & Y_{r}+Z_{r} \\
X_{b} & Y_{b}+Z_{b}
\end{array}\right)
\end{array}\right), \\
& M_{12}=a_{g} \operatorname{det}\left(\begin{array}{ll}
G_{i} & \operatorname{det}\left(\begin{array}{cc}
X_{b} & Y_{b}+Z_{b} \\
X_{r} & Y_{r}+Z_{r}
\end{array}\right) \\
B_{i} & \operatorname{det}\left(\begin{array}{ll}
X_{r} & Y_{r}+Z_{r} \\
X_{g} & Y_{g}+Z_{g}
\end{array}\right)
\end{array}\right)+a_{r} \operatorname{det}\left(\begin{array}{ll}
R_{i} & \operatorname{det}\left(\begin{array}{ll}
X_{g} & Y_{g}+Z_{g} \\
X_{b} & Y_{b}+Z_{b}
\end{array}\right) \\
B_{i} & \operatorname{det}\left(\begin{array}{ll}
X_{r} & Y_{r}+Z_{r} \\
X_{g} & Y_{g}+Z_{g}
\end{array}\right)
\end{array}\right), \\
& M_{13}=a_{b} \operatorname{det}\left(\begin{array}{cc}
G_{i} & \operatorname{det}\left(\begin{array}{ll}
X_{b} & Y_{b}+Z_{b} \\
X_{r} & Y_{r}+Z_{r}
\end{array}\right) \\
B_{i} & \operatorname{det}\left(\begin{array}{ll}
X_{r} & Y_{r}+Z_{r} \\
X_{g} & Y_{g}+Z_{g}
\end{array}\right)
\end{array}\right)+a_{r} \operatorname{det}\left(\begin{array}{ll}
R_{i} & \operatorname{det}\left(\begin{array}{cc}
X_{b} & Y_{b}+Z_{b} \\
X_{g} & Y_{g}+Z_{g}
\end{array}\right) \\
G_{i} & \operatorname{det}\left(\begin{array}{ll}
X_{r} & Y_{r}+Z_{r} \\
X_{b} & Y_{b}+Z_{b}
\end{array}\right)
\end{array}\right), \\
& M_{21}=a_{r} \operatorname{det}\left(\begin{array}{cc}
B_{i} & \operatorname{det}\left(\begin{array}{ll}
X_{r} & Y_{r}+Z_{r} \\
X_{g} & Y_{g}+Z_{g}
\end{array}\right) \\
R_{i} & \operatorname{det}\left(\begin{array}{ll}
X_{g} & Y_{g}+Z_{g} \\
X_{b} & Y_{b}+Z_{b}
\end{array}\right)
\end{array}\right)-a_{g} \operatorname{det}\left(\begin{array}{ll}
G_{i} & \operatorname{det}\left(\begin{array}{ll}
X_{b} & Y_{b}+Z_{b} \\
X_{r} & Y_{r}+Z_{r}
\end{array}\right) \\
B_{i} & \operatorname{det}\left(\begin{array}{ll}
X_{r} & Y_{r}+Z_{r} \\
X_{g} & Y_{g}+Z_{g}
\end{array}\right)
\end{array}\right),
\end{aligned}
$$




$$
\begin{aligned}
& M_{23}=a_{b} \operatorname{det}\left(\begin{array}{cc}
B_{i} & \operatorname{det}\left(\begin{array}{ll}
X_{r} & Y_{r}+Z_{r} \\
X_{g} & Y_{g}+Z_{g}
\end{array}\right) \\
R_{i} & \operatorname{det}\left(\begin{array}{ll}
X_{g} & Y_{g}+Z_{g} \\
X_{b} & Y_{b}+Z_{b}
\end{array}\right)
\end{array}\right)+a_{g} \operatorname{det}\left(\begin{array}{ll}
R_{i} & \operatorname{det}\left(\begin{array}{ll}
X_{b} & Y_{b}+Z_{b} \\
X_{g} & Y_{g}+Z_{g}
\end{array}\right) \\
G_{i} & \operatorname{det}\left(\begin{array}{ll}
X_{r} & Y_{r}+Z_{r} \\
X_{b} & Y_{b}+Z_{b}
\end{array}\right)
\end{array}\right), \\
& M_{31}=-a_{r} \operatorname{det}\left(\begin{array}{ll}
R_{i} & \operatorname{det}\left(\begin{array}{ll}
X_{b} & Y_{b}+Z_{b} \\
X_{g} & Y_{g}+Z_{g}
\end{array}\right) \\
G_{i} & \operatorname{det}\left(\begin{array}{ll}
X_{r} & Y_{r}+Z_{r} \\
X_{b} & Y_{b}+Z_{b}
\end{array}\right)
\end{array}\right)-a_{b} \operatorname{det}\left(\begin{array}{ll}
G_{i} & \operatorname{det}\left(\begin{array}{ll}
X_{b} & Y_{b}+Z_{b} \\
X_{r} & Y_{r}+Z_{r}
\end{array}\right) \\
B_{i} & \operatorname{det}\left(\begin{array}{ll}
X_{r} & Y_{r}+Z_{r} \\
X_{g} & Y_{g}+Z_{g}
\end{array}\right)
\end{array}\right), \\
& M_{32}=a_{b} \operatorname{det}\left(\begin{array}{ll}
R_{i} & \operatorname{det}\left(\begin{array}{ll}
X_{g} & Y_{g}+Z_{g} \\
X_{b} & Y_{b}+Z_{b}
\end{array}\right) \\
B_{i} & \operatorname{det}\left(\begin{array}{ll}
X_{r} & Y_{r}+Z_{r} \\
X_{g} & Y_{g}+Z_{g}
\end{array}\right)
\end{array}\right)-a_{g} \operatorname{det}\left(\begin{array}{ll}
R_{i} & \operatorname{det}\left(\begin{array}{ll}
X_{b} & Y_{b}+Z_{b} \\
X_{g} & Y_{g}+Z_{g}
\end{array}\right) \\
G_{i} & \operatorname{det}\left(\begin{array}{ll}
X_{r} & Y_{r}+Z_{r} \\
X_{b} & Y_{b}+Z_{b}
\end{array}\right)
\end{array}\right), \\
& K_{1}=-\left(a_{r} R_{i}+a_{g} G_{i}+a_{b} B_{i}\right) \operatorname{det}\left(\begin{array}{ll}
G_{i} & \operatorname{det}\left(\begin{array}{cc}
Y_{b} & X_{b}+Z_{b} \\
Y_{r} & X_{r}+Z_{r}
\end{array}\right) \\
B_{i} & \operatorname{det}\left(\begin{array}{ll}
Y_{r} & X_{r}+Z_{r} \\
Y_{g} & X_{g}+Z_{g}
\end{array}\right)
\end{array}\right), \\
& K_{2}=-\left(a_{r} R_{i}+a_{g} G_{i}+a_{b} B_{i}\right) \operatorname{det}\left(\begin{array}{ll}
B_{i} & \operatorname{det}\left(\begin{array}{cc}
Y_{r} & X_{r}+Z_{r} \\
Y_{b} & X_{b}+Z_{b}
\end{array}\right) \\
R_{i} & \operatorname{det}\left(\begin{array}{ll}
Y_{g} & X_{g}+Z_{g} \\
Y_{b} & X_{b}+Z_{b}
\end{array}\right)
\end{array}\right), \\
& K_{3}=\left(a_{r} R_{i}+a_{g} G_{i}+a_{b} B_{i}\right) \operatorname{det}\left(\begin{array}{ll}
R_{i} & \operatorname{det}\left(\begin{array}{cc}
Y_{b} & X_{b}+Z_{b} \\
Y_{g} & X_{g}+Z_{g}
\end{array}\right) \\
G_{i} & \operatorname{det}\left(\begin{array}{ll}
Y_{r} & X_{r}+Z_{r} \\
Y_{b} & X_{b}+Z_{b}
\end{array}\right)
\end{array}\right), \\
& N_{12}=a_{g} \operatorname{det}\left(\begin{array}{cc}
G_{i} & \operatorname{det}\left(\begin{array}{ll}
Y_{b} & X_{b}+Z_{b} \\
Y_{r} & X_{r}+Z_{r}
\end{array}\right) \\
B_{i} & \operatorname{det}\left(\begin{array}{ll}
Y_{r} & X_{r}+Z_{r} \\
Y_{g} & X_{g}+Z_{g}
\end{array}\right)
\end{array}\right)+a_{r} \operatorname{det}\left(\begin{array}{ll}
R_{i} & \operatorname{det}\left(\begin{array}{cc}
Y_{g} & X_{g}+Z_{g} \\
Y_{b} & X_{b}+Z_{b}
\end{array}\right) \\
B_{i} & \operatorname{det}\left(\begin{array}{ll}
Y_{r} & X_{r}+Z_{r} \\
Y_{g} & X_{g}+Z_{g}
\end{array}\right)
\end{array}\right), \\
& N_{13}=a_{b} \operatorname{det}\left(\begin{array}{cc}
G_{i} & \operatorname{det}\left(\begin{array}{cc}
Y_{b} & X_{b}+Z_{b} \\
Y_{r} & X_{r}+Z_{r}
\end{array}\right) \\
B_{i} & \operatorname{det}\left(\begin{array}{ll}
Y_{r} & X_{r}+Z_{r} \\
Y_{g} & X_{g}+Z_{g}
\end{array}\right)
\end{array}\right)+a_{r} \operatorname{det}\left(\begin{array}{ll}
R_{i} & \operatorname{det}\left(\begin{array}{cc}
Y_{b} & X_{b}+Z_{b} \\
Y_{g} & X_{g}+Z_{g}
\end{array}\right) \\
G_{i} & \operatorname{det}\left(\begin{array}{ll}
Y_{r} & X_{r}+Z_{r} \\
Y_{b} & X_{b}+Z_{b}
\end{array}\right)
\end{array}\right),
\end{aligned}
$$




$$
\begin{aligned}
& N_{21}=a_{r} \operatorname{det}\left(\begin{array}{ll}
B_{i} & \operatorname{det}\left(\begin{array}{cc}
Y_{r} & X_{r}+Z_{r} \\
Y_{g} & X_{g}+Z_{g}
\end{array}\right) \\
R_{i} & \operatorname{det}\left(\begin{array}{ll}
Y_{g} & X_{g}+Z_{g} \\
Y_{b} & X_{b}+Z_{b}
\end{array}\right)
\end{array}\right)-a_{g} \operatorname{det}\left(\begin{array}{ll}
G_{i} & \operatorname{det}\left(\begin{array}{cc}
Y_{b} & X_{b}+Z_{b} \\
Y_{r} & X_{r}+Z_{r}
\end{array}\right) \\
B_{i} & \operatorname{det}\left(\begin{array}{ll}
Y_{r} & X_{r}+Z_{r} \\
Y_{g} & X_{g}+Z_{g}
\end{array}\right)
\end{array}\right), \\
& N_{23}=a_{b} \operatorname{det}\left(\begin{array}{ll}
B_{i} & \operatorname{det}\left(\begin{array}{ll}
Y_{r} & X_{r}+Z_{r} \\
Y_{g} & X_{g}+Z_{g}
\end{array}\right) \\
R_{i} & \operatorname{det}\left(\begin{array}{ll}
Y_{g} & X_{g}+Z_{g} \\
Y_{b} & X_{b}+Z_{b}
\end{array}\right)
\end{array}\right)+a_{g} \operatorname{det}\left(\begin{array}{ll}
R_{i} & \operatorname{det}\left(\begin{array}{ll}
Y_{b} & X_{b}+Z_{b} \\
Y_{g} & X_{g}+Z_{g}
\end{array}\right) \\
G_{i} & \operatorname{det}\left(\begin{array}{ll}
Y_{r} & X_{r}+Z_{r} \\
Y_{b} & X_{b}+Z_{b}
\end{array}\right)
\end{array}\right), \\
& N_{31}=-a_{r} \operatorname{det}\left(\begin{array}{ll}
R_{i} & \operatorname{det}\left(\begin{array}{ll}
Y_{b} & X_{b}+Z_{b} \\
Y_{g} & X_{g}+Z_{g}
\end{array}\right) \\
G_{i} & \operatorname{det}\left(\begin{array}{ll}
Y_{r} & X_{r}+Z_{r} \\
Y_{b} & X_{b}+Z_{b}
\end{array}\right)
\end{array}\right)-a_{b} \operatorname{det}\left(\begin{array}{ll}
G_{i} & \operatorname{det}\left(\begin{array}{cc}
Y_{b} & X_{b}+Z_{b} \\
Y_{r} & X_{r}+Z_{r}
\end{array}\right) \\
B_{i} & \operatorname{det}\left(\begin{array}{ll}
Y_{r} & X_{r}+Z_{r} \\
Y_{g} & X_{g}+Z_{g}
\end{array}\right)
\end{array}\right), \\
& N_{32}=a_{b} \operatorname{det}\left(\begin{array}{cc}
R_{i} & \operatorname{det}\left(\begin{array}{cc}
Y_{g} & X_{g}+Z_{g} \\
Y_{b} & X_{b}+Z_{b}
\end{array}\right) \\
B_{i} & \operatorname{det}\left(\begin{array}{ll}
Y_{r} & X_{r}+Z_{r} \\
Y_{g} & X_{g}+Z_{g}
\end{array}\right)
\end{array}\right)-a_{g} \operatorname{det}\left(\begin{array}{ll}
R_{i} & \operatorname{det}\left(\begin{array}{cc}
Y_{b} & X_{b}+Z_{b} \\
Y_{g} & X_{g}+Z_{g}
\end{array}\right) \\
G_{i} & \operatorname{det}\left(\begin{array}{ll}
Y_{r} & X_{r}+Z_{r} \\
Y_{b} & X_{b}+Z_{b}
\end{array}\right)
\end{array}\right) .
\end{aligned}
$$

Analysis of the matrix equations (15) shows that the coefficients $M_{i j}, N_{i j}$, are symmetric about zero main diagonals, are equal in magnitude and opposite sign: $M_{12}=-M_{21}, M_{13}=-M_{31}, M_{23}=-M_{32}, N_{12}=-N_{21}$, $N_{13}=-N_{31}, N_{23}=-N_{32}$. It is possible to show that such matrices have determinant equal to zero. Thus, according to the rule of Cramer in equations (15), there are theoretically an infinite number of solutions (extreme points) on a continuum set. The addition of these matrices corresponding columns free members $L_{1}-L_{3}$ и $K_{1}-K_{3}$ and the calculation of the rank the original and augmented matrices leads to the conclusion about incompatibility equations of both matrix systems.

\section{Automated Workstation for Researches}

Assessment of color coordinates of display elements indicated on LCD screen is carried out by means of source computer and computer assisted design (CAD) forming automated workstation (AWS).

AWS CAD includes

1. CAD software comprising of data components, functional software (FSW) components and test software (TSW) components, application software for data and programs uploading into indicator. Software components are programs' texts and data codes documents. Data components are

- palette ( $R G B$ colors decimal code table used by software engineers when creating indicative frames of avionics, which include displaying navigation parameters and geoinformation data);

- type and symbol library of coordinated configuration represented in indicator's graphics controller memory in vector and raster form for different by size (in pixels) character locations (the library includes coordinated configuration characters and symbols images in different languages - Russian, Latin alphabet, etc., Roman and Arabic numerals, etc.);

- internal angle library (increments calculated by trigonometric functions for graphic primitives construction in indicator screen such as arc and circle using approximation by inscribed polygon).

CAD software allows operator to solve the following tasks using automated method:

- in source computer - to create and edit files of data uploading components used when displaying indicative 
frames;

- to enter data uploading components from source computer into the memory of graphics controller using mechanical interface;

- to enter FSW and TSW components from source computer into the memory of computer module for performance evaluation in all working modes.

2. CAD mathematical support including complex of mathematical methods and quality criteria, mathematical models and computer-aided engineering algorithms necessary for process optimization of color palette selection by operator. CAD mathematical support allows operator to solve the following tasks:

- to select methods and criteria appropriate for colour palette selection optimization procedure;

- to conduct mathematical analysis of data resulting from luminance measurement and image luminance difference assessment for the purpose of search for colours and shades component, which have augmented perceptual data for man.

3. CAD information support including complex of data necessary for automated selection procedures. The base of CAD information support is automated data banks, which consist of different CAD databases and DBMS. AWS CAD information support includes

- standards, regulations and specifications (in particular, avionic equipment certification guidance 25-11A), national and industrial standards for display medium, standards for documents execution, design specifications;

- current (previously obtained by other engineers) standard design solutions for indicators development with an indication of significant for election procedure products' parameters;

- existing image coding systems (mathematical relations, binding codes, colour coordinates, and colours and shades wavelengths).

CAD information support allows operator to solve the following tasks:

- select relevant for avionics subsets of colours and shades used when displaying navigation parameters and geoinformation data from the complete set of colours and shades $\left(2^{18}\right.$ for 6-bit LCD screen or $2^{24}$ for 8-bit LCD screen) potentially displayable in indicator;

- to select picture coding system wherein chromaticity desired coordinates selection procedure will be carried out;

- to select picture coding system wherein physical device - graphical controller - engineering will be carried out;

- to select picture coding system wherein physical device software engineering will be carried out.

4. CAD technical support including the complex of interrelated and interactive technical aids designed for colour palette selection process optimization. CAD technical support allows operator to solve the following tasks:

- preparation of data components, FSW and TSW components. CAD technical support designed for this task solution comprises of source computer. Source computer with installed software allows operator to carry out procedures of preparation of automation and editing of data components and programs' components before their entering into indicator using program method. Source computer makes it possible to code uploading components, save data and programs in source computer hard drive and edit previously created data;

- data communication. CAD technical support group designed for this task solution consists of source computer, communication cables and power sources. The group allows operator to enter data into indicator, visually and apparatus control entry entirety of control totals values data;

- program data processing. CAD technical support group designed for this task solution consists of source computer; lighting stand, including digital photometer, lucimeter (or chromometer), line autotransformer, external illumination lamps and LCD screen blower; production equipment and communication cables; power supply sources. The group allows operator to measure screen light intensity in any colour (shade), including background (black, grey), transfer LCD screen luminance measurement results into source computer via mechanical interface, calculate luminance difference of display elements in screen under exposure of external illumination from 0 kilolux to 75 kilolux;

- data display and documentation. CAD technical support group designed for this task solution consists of source computer and printer. The group allows operator to timely present and document obtained results (design solutions) in terms of colour palette components selection, prepare research and development report 
concerning findings. The group allows operator to change his performance on AWS due to the use of source compute of different types by means of multiprogram and interactive operation of application software installed in source computer;

- ready-made design solutions archivation support. CAD technical support designed for this task solution consists of source computer. Source computer allows operator to save obtained in the process of research components of colour palette for different models of indicators made based on LCD screens of different manufacturing companies under exposure to the various levels of external illumination.

\section{Conclusion}

The analysis of the system (6) shows that changing the decimal code of the one color component, for example, red $R$, when zero code values of the other two colors $(G$ and $B)$ do not displace $(x, y)$ chromaticity coordinates on the $X Y$-plane - the determinants in the numerator of (6) in this case is equal to zero. Red color with code $R=5$, $R=120, R=200, R=255$ and others when $G=0$ и $B=0$ have the equal chromaticity with $(x, y)$ coordinates on $X Y$-plane. In a similar $G$ and $B$. While increasing code values $R G B$ in equal proportions in all three components of the primary colors offset $(x, y)$ coordinates also does not occur, because these changes correspond to one color - white. Offset $(x, y)$ chromaticity coordinates on the $X Y$-plane by the amount $(\Delta x, \Delta y)$ formed when «mixing» of the components of the three primary colors in different proportions.

Also the analysis of the system (6) and the results of computations based on it, show that value of the discrete increments $\Delta x, \Delta y$ distributed unevenly on $X Y$-plane, step change $(x, y)$ chromaticity coordinates within the various zones for color's shades (Figure 1,c) when changing $R G B$ codes on the unit is different. The uneven distribution of the increments on field caused, primarily, because of nonlinearity of the transformation (2).

The estimated increments of equations of system (6) show that the absolute values of the majority of couples increments $\Delta x, \Delta y$ not equal, Grassmann's transformation resolution on $x$-coordinate in general not equal on $y$ - coordinate, so when choosing a colorimeter the choice of metrological characteristics of the measuring instrument should be based on the minimum of two values: $\min (\Delta x, \Delta y)$, obtained by changing the $R G B$ code in the entire range of acceptable values.

Obtained in the calculation process numeric values of Grassmann's transformation resolution:

- horizontal coordinate of the minimum $|\Delta x|=8,45 \cdot 10^{-10}$;

- vertical coordinate of the minimum $|\Delta y|=7,23 \cdot 10^{-10}$;

- diagonally of the minimum $\sqrt{\Delta x^{2}+\Delta y^{2}}=1,614 \cdot 10^{-6}$,

meet a CIE 1931 (standard colorimetric observer) with the evaluation system white balance D-65 and with the adopted coding system $« 8$ bits per primary color component». Equation system (6) is given in analytical form (the dependence of the increments from the coefficients of the matrix CIE) and can used for the estimation of grassmann's transformation resolution such as for CIE 1931 as for CIE 1964 г. with different systems for the assessment of white balance: D-75, D-65, D-55, D-50 and etc., within each of which the components of the primary colors $X_{r}, X_{g}, X_{b}, Y_{r}, Y_{g}, Y_{b}, Z_{r}, Z_{g}, Z_{b}$ of basic transformations (1) are different.

It should also be noted that the numerical values of Grassmann's transformation resolution on several orders of magnitude less technical characteristics of existing instrumentation. In this regard, in experiments on the assessment of the visual characteristics of the perception of color palette significant number of color transitions from one shade to another cannot be determined by modern tools of measurement with the required avionics precision.

\section{References}

Barber, S. et al. (2008). US Patent 7,417,641 B1: Aeronautical chart display apparatus and method.

Evanicky, D., Granger, Ed., Ingulsrud, J., \& Meng, A. T. (2009). US Patent 2009/0051711 A1: Compact flat panel color calibration system, Feb. 26.

Ibraheem, N. A., Hasan, M. M., Khan, R. Z., \& Mishra, P. K. (2012). Understanding color models: A Review. ARPN Journal of Science and Technology, 2(3), 265-275.

Ishihara, K., \& Kadota, Sh. (2009). US Patent 7,492,495 B2: Optical scanning apparatus and scanning image display apparatus, Feb.17.

Kamoto, S. et al. (2011). US Patent 7,982,384 B2: Image display device and light emission device.

Kumar, S. V., \& Ramana, P. V. (2014). Color selection algorithm design for smart lighting application. 
International journal of computer science and information technology \& security, 4(1), 8-13.

Menesatti, P., Angelini, C., Pallottino, F., Antonucci, F., Aguzzi, Y., \& Costa, C. (2012). RGB color calibration for quantitative image analysis: The "3D thin-plate alpine" warping approach. Sensors, 12, 7063-7079. http://dx.doi.org/10.3390/s120607063

Schanda, J. (2007). Colorimetry Understanding the CIE System. Wiley-Interscience John Wiley \& Sons, INC., Publication, 499.

Seetzen, H., Makki, S., Ip, H., Wan, Th., Kwong, V., Ward, Gr., Heidrich, W., \& Whitehead, L. (2007). Self-calibrating wide color gamut high dynamic range display. Proceeding of SPIE-IS\&T Electronic Imaging, vol.6492, 64920Z, 1-9. http://dx.doi.org/10.1117/12.720875

Yamashita, J., \& Uchino, K. (2014). U. S. Patent 8,872,740 B2: Display Apparatus and Display Apparatus Driving Method.

Yoshida, Y., \& Kimura, H. (2008). U. S. Patent 2008/0180385 A1: Liquid Crystal Display Device and Driving Method Thereof.

Zargaryants, G. S., \& Mikhailov, O. M. (2008). Integral remote colorimeter bases on the RGB colorimetric system. Light \& Engineering, 16(3), 69-77.

\section{Copyrights}

Copyright for this article is retained by the author(s), with first publication rights granted to the journal.

This is an open-access article distributed under the terms and conditions of the Creative Commons Attribution license (http://creativecommons.org/licenses/by/3.0/). 